\title{
Planet 1894 BO (Roberts April 9).
}

Auf einer photographischen Aufnahme des Nebels H I. 143, welche Mr. J. Roberts am 20 zöll. Reflector bei $3^{\mathrm{h}} 2^{\mathrm{m}}$ Expositionszeit am 9. April 1894 gemacht hat, befindet sich ein von einem schwachen Planeten gezogener Bahnstrich (vgl. Monthl. Not. LV. 14). Nach Mittheilungen von Herrn Prof. Downing ist der Planetenort für April 9 $12^{\mathrm{b}}$ M. Z. Greenw.

$$
a=\mathrm{r}^{\mathrm{h}} 59^{\mathrm{m}} 8^{\mathrm{s}} \quad \delta=+3^{\circ} 8^{\prime}
$$

Die länge des Striches ist $71^{\prime \prime}$, der Positionswinkel $3 \circ 3^{\circ} 4^{\circ}$. Hieraus würde eine tägliche Bewegung

$$
\mathrm{d} a=-3^{2^{\mathrm{s}}} \quad \mathrm{d} \delta=+5.2
$$

folgen. Leider haben weder Herr Charlois noch Herr Wolf um jene Zeit die Planetengegend aufgenommen, so dass ïber die Bahn des offenbar neuen Planeten sich kaum etwas sagen lässt. Nur das sieht man, dass die Breiten-

bewegung positiv war, dass also der aufsteigende Knoten zwischen $140^{\circ}$ und $170^{\circ}$ liegen mag. Herr Wolf machte mich noch besonders darauf aufmerksam, dass bei solch schwachen Planeten die Striche erheblich zu kurz ausfallen. In wie weit die Lichtstärke des 20 zöll. Reflectors diesen Nachtbeil ausgleicht, lässt sich nicht angeben, da kein anderer bekannter Planet auf derselben Platte steht und die Heranziehung anderer Aufnahmen, die unter anderen Verhältnissen gemacht sind, für den vorliegenden speciellen Fall nichts nützen kann. Obige Bewegung ist allerdings sehr klein; der Planet müsste daher sehr weit entfernt gewesen sein oder bei mässiger Entfernung nahe dem Perihel seiner dann recht excentrisch anzunehmenden Bahn gestanden haben.

Vielleicht tragen diese Erwägungen dazu bei, den Planeten identificiren zu können, falls in den nächsten Jahren seine Wiederentdeckung gelingen sollte.

Beriin, Kgl. Recheninstitut, 1894 Dec. 29.

A. Berberich.

\section{Bitte um Mittheilung von Beobachtungen der Planeten 1894 BD, BF und BG.}

Von obigen drei Planeten sind mir directe Beobachtungen bisher noch nicht zugegangen. Ich wäre den Herren Beobachtern, welche solche erhalten haben, dankbar, wenn sie mir dieselben bald mittheilen wollten. Die Heidelberger photographischen Positionen sind für die Bahnberechnung nicht güstig vertheilt. Für den recht schwachen Planeten 1894 BD war es mir nur möglich, die folgende Kreisbahn aus Nov, 1 und 7 (Heidelberg photogr.) zu rechnen.

Berlin, Kgl. Recheninstitut, 1894 Dec. 30.
Planet I $894 \mathrm{BD}$.

Epoche 1894 Nov. 7.5 M. Z. Berlin.

$$
\begin{aligned}
& u=336^{\circ} 47: 8 \\
& \delta=639.1 \quad 1894.0 \\
& i=53^{6.2} \\
& \mu=988 \text { ". 1 } 4 \\
& \log a=0.37012
\end{aligned}
$$

\section{Osservazione della Cometa 1894 ... (E. Swift) \\ al I 5.5 inch di Cooke in Teramo \\ $\left(\varphi=+42^{\circ} 39^{\prime} 27^{\prime \prime}\right.$. Riduzione dei tempi a Berlino $-1^{\mathrm{m}} 2 \mathrm{I}^{\mathrm{s}}$.)}

1894 Dic. $277^{\mathrm{h}} 37^{\mathrm{m}} 34^{\mathrm{s}}$ t. m. Teramo $\Delta \alpha=+\mathrm{I}^{\mathrm{m}} 19^{\mathrm{s}} \cdot 45 \quad \Delta \delta=-9^{\prime} 8^{\prime \prime} .0 \quad$ Cfr. 10 $\alpha$ app. $=0^{\mathrm{h}} 0^{\mathrm{m}} 11^{\mathrm{s}} \cdot 64(9 \cdot 344)$

$\delta$ app. $=-0^{\circ} 44^{\prime} 4 I^{\prime \prime} \cdot 5(0.781)$.

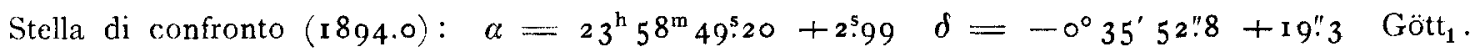

Pare rotonda, con diametro di circa $I^{\prime}$.

A. Berberich.

\section{Literarische Anzeigen.}

Francesco Porro. Astronomia sferica. Roma 1 894 . 136 S. $8^{0}$.

J. H. Lambert. Anmerkungen und Zusätze zur Entwerfung der Land- und Himmelskarten. Herausgegeben von A. Wangerin. Leipzig I894. Wilhelm Engelmann. (Ostwald's Klassiker der exakten Wissenschaften. Nr. 54.)

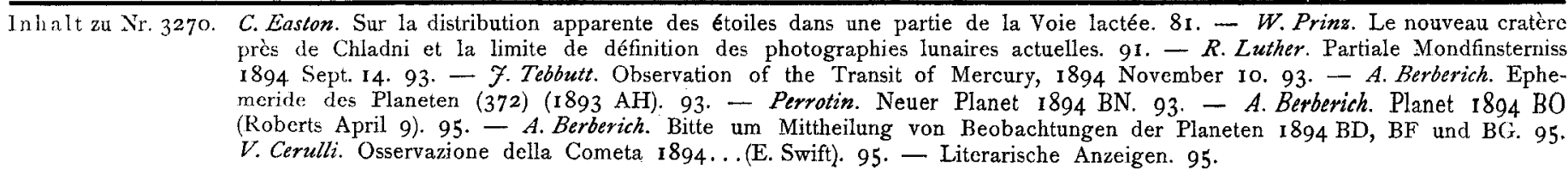
Geschlossen 1895 Jan. ro. Heransgeber: A. Krueger. Druck von C. Schaidt. Expedition: Sternwarte in Kiel. 